\title{
A Heavyweight Merger
}

\author{
The heaviest black hole merger detected to date provides evidence for a \\ progenitor black hole in a theoretically forbidden gap and for a resulting \\ black hole in the long-sought intermediate mass range.
}

By Rosalba Perna

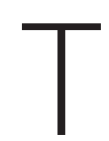

he observation of gravitational waves has opened a new window on the Universe, providing the first direct proof of the existence of black holes as described by general relativity [1]. With ten black hole mergers confirmed since 2015, these observations have revealed black holes with a large range of masses and with different spins. Now, the LIGO Collaboration and the Virgo Collaboration have reported the detection of the heaviest black hole merger to date-a 65-solar-mass black hole and an 85-solar-mass black hole coalescing to form a 142-solar-mass black hole (Fig. 1) [2, 3]. Two aspects make this event exceptional. First, the size of the larger progenitor (85 solar masses) falls in a "gap" where black holes are not expected to form by conventional mechanisms. Second, the detection of the 142-solar-mass remnant is the first direct

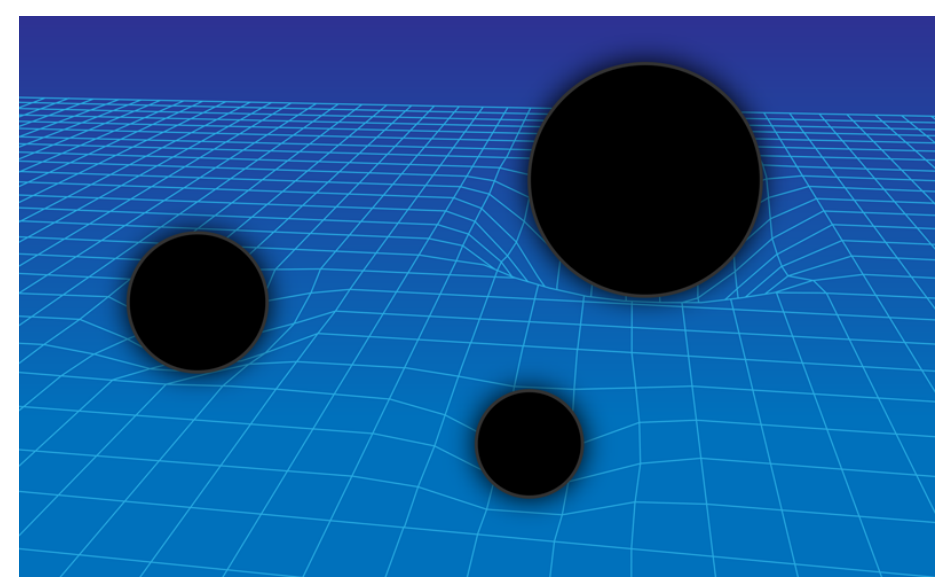

Figure 1: The LIGO and Virgo Collaborations have detected the gravitational waves released by the heaviest merger ever documented-a 65-solar-mass black hole and an 85-solar-mass black hole merging into a 142-solar-mass black hole.

Credit: APS/Alan Stonebraker observation of an intermediate-mass black hole (IMBH)-a class of black holes with masses in between about one hundred and tens of thousands of solar masses.

According to Einstein's equations, black holes can have any mass. However, in our Universe black holes of a certain mass can only exist if there is an astrophysical process for creating them. The collapse of massive stars provides one key pathway to black hole formation that sets some constraints on expected black hole masses.

A stellar-mass black hole is formed when a star dies in a dramatic supernova explosion. The explosion occurs once nuclear fusion has turned most of the star's core into iron. At this point, the star is out of nuclear fuel, and its core begins to collapse onto itself. If the star mass is larger than a certain value (estimated to be at least 2.17 solar masses [4]), its core is doomed to collapse to a black hole. Black holes formed in this way can have a wide range of masses but only up to a maximum value set by so-called pair instability [5].

Pair instability is a phenomenon that drains a star's energy through the production of electron-positron pairs. In a hot star, the core produces gamma rays that exert "photon pressure" on the outer stellar layers, supporting them against the gravitational pull. If the star core is larger than about 65 solar masses, however, the gamma rays efficiently convert into electron-positron pairs, and this photon support wanes. The outer layers then collapse inwards, the nuclear burning accelerates in a runaway fashion, and the star blows apart without leaving behind any black hole remnant. This picture holds up to about 135 solar masses, beyond which the star directly collapses into a black hole. The instability thus creates a mass gap-between $\sim 65$ and $\sim 135$ solar masses, where 
black holes aren't theoretically expected [6]. Until now, none of the progenitor black holes detected by LIGO and Virgo lay within this gap [1].

Other black hole observations have uncovered a totally different weight class. At the high end of the mass spectrum, there's evidence for "supermassive" black holes weighing from about one hundred thousand to billions of solar masses. Examples include the black hole at the Milky Way center [7] and that at the center of the M87 galaxy recently imaged by the Event Horizon Telescope [8]. IMBHs-lighter than supermassive black holes but heavier than stellar-mass black holes-are also thought to be able to form via several mechanisms. While several candidates have been identified among so-called ultraluminous $x$-ray sources, no IMBH has been directly observed to date.

The new merger detection, dubbed GW190521, thus delivers two remarkable results [8]. The 142-solar-mass remnant proves that IMBHs do exist. And the 85 -solar-mass progenitor shows that black holes can, surprisingly, have masses within the pair-instability gap. These findings might have important implications for our understanding of astrophysical black hole formation.

Two probable scenarios could lead to a black hole binary like the one detected. In the first scenario, the black holes in the binary result from the collapse of two stars in a binary system. This would mean that the 85 -solar-mass black hole is directly formed from a star in the forbidden gap. If this is the case, the result could be explained by revising our models for nuclear reactions in stellar cores. Recent calculations [9] have shown that the lower boundary of the gap is sensitively dependent on the rate of a specific nuclear reaction, which burns carbon-12, turning it into oxygen-16. If this rate is smaller than assumed by current models, the mass gap would shift toward larger masses, which could accommodate the measured value.

In the second scenario, the most massive black hole would be a "second generation" black hole, resulting from the merger of two smaller black holes. The black hole binary would thus result not from a stellar binary but from dynamical gravitational interactions that bring the black holes together. The spins of the merging black holes could help in distinguishing the two scenarios, since they are expected to be roughly aligned in the case of an isolated binary evolution and to be randomly oriented in a dynamical interaction scenario. The researchers' analysis shows the spins of GW190521's merging black holes are likely misaligned [3]. This result mildly favors the second scenario, but the evidence isn't conclusive.

Further hints may come from the recent identification of the possible electromagnetic counterpart of this merger. From the same region of the sky as GW190521, the Zwicky Transient Facility (ZTF) detected a flare emerging 35 days after the gravitational-wave signal (see Focus: Possible Flare from Black Hole Merger) [10]. This flare could have been produced by the gas in the black holes' environment, which gets heated by shock waves induced by the merger. If the connection between flare and merger is true, then the merger occurred in the disk of an active galactic nucleus. In this environment, which is conducive to multiple-generation black holes weighing more than 50 solar masses [11], the presence of a black hole within the mass gap wouldn't be surprising.

In the coming years, LIGO's and Virgo's detectors will likely see more merger events of this heavier kind (see also Trend: Gravitational-Wave Astronomy Still in Its Infancy). Better statistics on the population of IMBHs may shed light on the cosmological growth of supermassive black holes, which are thought to be assembled from IMBH building blocks. And with more events involving black holes with masses within and around the theoretically forbidden gap, it may be possible to settle questions on formation scenarios and on the key nuclear reactions that set the range of achievable masses.

Rosalba Perna: Department of Physics and Astronomy, Stony Brook University, Stony Brook, NY, USA

\section{REFERENCES}

1. B. P. Abbott et al. (LIGO Scientific Collaboration and Virgo Collaboration), "Observation of gravitational waves from a binary black hole merger," Phys. Rev. Lett. 116, 061102 (2016); B. P. Abbott et al. (LIGO Scientific Collaboration and Virgo Collaboration), "Binary black hole population properties inferred from the first and second observing runs of advanced LIGO and advanced Virgo," Astrophys. J. Lett. 882, 24 (2019).

2. R. Abbott et al. (LIGO Scientific Collaboration and Virgo Collaboration), "GW190521: A binary black hole merger with a total mass of $150 M_{\odot}$," Phys. Rev. Lett. 125, 101102 (2020).

3. B. P. Abbott et al. (LIGO Scientific Collaboration and Virgo 
Collaboration), "Properties and astrophysical implications of the $150 M_{\odot}$ binary black hole merger GW90521," Astrophys. J. Lett. 900, L13 (2020).

4. H. T. Cromartie et al., "Relativistic Shapiro delay measurements of an extremely massive millisecond pulsar," Nat. Astron. 4, 72 (2019).

5. S. E. Woosley, "Pulsational pair-instability supernovae," Astrophys. J. 836, 244 (2017).

6. M. Mapelli et al., "Impact of the rotation and compactness of progenitors on the mass of black holes," Astrophys. J. 888, 76 (2020).

7. A. M. Ghez et al., "Measuring distance and properties of the Milky Way's central supermassive black hole with stellar orbits," Astrophys. J. 689, 1044 (2008).

8. K. Akiyama et al. (The Event Horizon Telescope Collaboration), "First M87 Event Horizon Telescope results. I. The shadow of the supermassive black hole," Astrophys. J. Lett. 875, 1 (2019).

9. R. Farmer et al., "Constraints from gravitational wave detections of binary black hole mergers on the ${ }^{12} \mathrm{C}(\alpha, \gamma){ }^{16} \mathrm{O}$ rate," arXiv:2006.06678.

10. M. J. Graham et al., "A candidate electromagnetic counterpart to the binary black hole merger gravitational-wave event S190521g," Phys Rev. Lett. 124, 251102 (2020).

11. Y. Yang et al., "Hierarchical black hole mergers in active galactic nuclei," Phys. Rev. Lett. 123, 181101 (2019). 